\title{
In this issue
}

\author{
Fred T. Bosman ${ }^{1}$
}

(C) Springer-Verlag Berlin Heidelberg 2016

The July issue opens with a review paper on the lymphatic circulation by Adamczyk et al. (DOI 10.1007/s00428-0161945-6). The topic is important and merits more attention, given the central role of lymphatics in a variety of pathological processes. Understanding the peripheral lymphatic circulation has been greatly helped by the development of antibodies against proteins specifically expressed on lymphatic endothelium, such as podoplanin, Lyve-1 and Prox-1. Such tools have facilitated understanding of the role the peripheral lymphatic vessels play in lymphatic spread of cancer but also in various forms of lymphoedema, inflammatory reactions and vascular malformations. The paper reviews the scientific data in depth and provides practical hints for better understanding the role of lymphatics in the pathophysiology of disease as well as diagnosis of diseases of lymphatic vessels or in which lymphatic vessels are implicated.

Giuliani et al. (DOI 10.1007/s00428-016-1940-y) revisit the association of expression/amplification of HER2 gene with prognosis in infiltrating breast cancer. They specifically asked two questions: what is the proportion of HER2 positive cases (immunohistochemistry score 3 and score 2 with HER2 amplification confirmed in a FISH test) and is there a difference between these two subgroups (e.g. HER2 3+ and HER2 $2+/$ FISH amplified)? The size of their cohort study is such that the results can be taken as relevant. In such a study old knowledge can be updated and as a consequence, with present day

Fred T. Bosman

fred.bosman@citycable.ch

1 Institute of Pathology, University Medical Center (CHUV) of Lausanne, Lausanne, Switzerland methodology, the proportion of HER2+ cases appeared to be lower than previously reported. Some old knowledge is confirmed in that ER, PgR, grade and pT appeared to be significantly associated with HER2 positive status. An interesting finding was that HER3+ cases were more frequently ER and PgR negative than HER2 2+/HER2 amplified cases and more frequently of higher grade and $\mathrm{T}$ stage. This provides evidence for intrinsic heterogeneity in HER2 positive breast cancer.

Ardighieri et al. address in their paper (DOI 10.1007 /s00428-016-1933-x) nature and origin of pelvic carcinosarcomas. These are rare aggressive biphasic tumors that occur in the ovary, fallopian tube or peritoneum and often bilateral. To investigate clonal relationships, the authors focused on p53 protein immunohistochemistry and TP53 gene mutations in a small cohort of these tumours. They also looked for accompanying serous tubal intraepithelial carcinoma, on the assumption that this might constitute a precursor lesion. Serous tubal intraepithelial carcinomas were indeed found in the majority of cases (notably when bilateral) but only when the carcinoma component of the carcinosarcoma had high grade serous features. Strikingly, p53 immunohistochemistry results were the same for the bilateral lesions and the tubal intraepithelial carcinomas and, even more striking, in these different compartments the same TP53 mutation was found. The authors interpret this as evidence for a clonal relation between these neoplastic lesions, which implies that bilateral pelvic carcinosarcomas are metastatic, and they contend that these tumours have an extra-ovarian origin in a serous tubal intraepithelial carcinoma.

Finally, Antic et al. (DOI 10.1007/s00428-016-1952-7) contribute to the continuing story of new subtypes of renal cell carcinoma. What used to be a single morphological (be it morphologically heterogeneous) category has meanwhile evolved into more than a handful of distinct subtypes, with 
different immunohistochemical characteristics and molecular properties, potentially requiring different targeted therapy approaches. The authors identified six histologically unusual renal cell carcinomas with a tubulopapillary architecture, composed of clear cells and a less than specific immunohistochemical profile. Of these cases a detailed molecular profile was established which could then be compared with that of renal cell carcinomas with typical morphological and or immunohistochemical features associated with a specific subtype (clear cell, clear cell papillary and MiT family translocation RCC). Four cases could be classified into one of the existing subtypes but two cases with monosomy of chromosome 8 could not and these were further studied by next generation sequencing. Although no new variants were detected, such studies may uncover additional subtypes which will contribute to the development of targeted therapy for renal cancer.

The cover image is taken from this paper and illustrates the loss of one copy of chromosome 8 in a genome wide copy number variation test. 